\title{
Uno sguardo agli interventi di messa in sicurezza per la bonifica dei siti contaminati
}

\author{
Ana Lucía Russo Rinaldi ${ }^{1}$ \\ ${ }^{1}$ Tecnologie per l'ambiente
}

\begin{abstract}
Gli interventi di messa in sicurezza, a differenza di quelli di bonifica che vanno ad eliminare la sorgente di contaminazione, hanno lo scopo di ridurre la mobilità degli inquinanti e la diffusione dei contaminanti dal sito all'ambiente mediante l'isolamento del sito stesso. Il presente lavoro affronta lo studio degli interventi di messa in sicurezza attraverso l'adozione di tecniche di ingegneria ambientale nell'ambito della normativa Italiana ed Inglese. A tal fine, verrà discusso il caso di un sito contaminato a Londra e verranno analizzate le principali tecniche di messa in sicurezza attualmente disponibili.
\end{abstract}

\section{Introduzione}

Per bonifica e ripristino ambientale si intendono tutti gli interventi che hanno l'obiettivo di ridurre la contaminazione del sito a livelli tali da non costituire rischio per la salute della popolazione e sono determinati dall'analisi di rischio sito specifica ${ }^{1}$, finché gli interventi di messa in sicurezza sono finalizzati all'isolamento delle fonti inquinanti, e al contenimento della diffusione degli inquinanti per impedirne il contatto con l'uomo e con i recettori ambientali circostanti.

Essi hanno carattere di:

Urgenza in caso di rilasci accidentali o di improvviso accertamento di una situazione di contaminazione o di pericolo di contaminazione.

Continuità e compatibilità con le lavorazioni svolte nei siti produttivi in esercizio.

- Definitività nei casi in cui, nei siti non interessati da attività produttive in esercizio, non sia possibile procedere alla rimozione degli inquinanti pur applicando le migliori tecnologie disponibili a costi sopportabili di cui all'allegato 3 alla parte IV del D. Lgs. 152/2006. 
La messa in sicurezza di un sito inquinato è comprensiva delle azioni di monitoraggio e controllo finalizzate alla verifica nel tempo delle soluzioni adottate ed il mantenimento dei valori di concentrazione degli inquinanti nelle matrici ambientali interessate al di sotto dei valori soglia di rischio (CSR). ${ }^{2}$

Ciò che riguarda agli interventi di messa in sicurezza dei siti inquinati ricade nel D. Lgs. 152/2006, Parte IV -Titolo V e definisce tre modalità di interventi di messa in sicurezza:

D'emergenza (art. 240, lett. m): interventi immediati o a breve termine, da mettere in opera nelle condizioni di emergenza in caso di eventi di contaminazione repentini di qualsiasi natura, atto a contenere la diffusione delle sorgenti primarie di contaminazione, impedirne il contatto con altre matrici presenti nel sito e a rimuoverle.

Operativa (art. 240, lett. $\mathrm{n}$ ): interventi eseguiti in un sito con attività in esercizio atti a garantire un adeguato livello di sicurezza per le persone e per l'ambiente.

- $\quad$ Permanente (art. 240, lett. o): interventi atti a isolare in modo definitivo le fonti inquinanti rispetto alle matrici ambientali circostanti e a garantire un elevato e definitivo livello di sicurezza per le persone e per l'ambiente.

D'altra parte, nello stesso decreto, I'Allegato 3 formula "Criteri generali per la selezione e l'esecuzione degli interventi di bonifica e ripristino ambientale, di messa in sicurezza (d'urgenza, operativa o permanente), nonché per l'individuazione delle migliori tecniche d'intervento a costi sopportabili", che propone di illustrare i criteri generali da seguire sia nella selezione che nell'esecuzione degli interventi di bonifica e ripristino ambientale, di messa in sicurezza d'urgenza, operativa, e permanente. Quindi, sono presentate le diverse opzioni da prendere in considerazione in quanto riguarda a un'effettiva eliminazione/riduzione della contaminazione e conseguire un'efficace azione di protezione delle matrici ambientali influenzate dagli effetti del sito mediante la messa in sicurezza dello stesso. Oltre a questo, i criteri minimi per l'esecuzione di interventi di isolamento superficiale vengono stabiliti nel D.lgs. 36/2003 relativa alle discariche di rifiuti, e anche lo spessore e conducibilità idraulica della barriera nei sistemi di isolamento perimetrale. $^{3}$

La definizione e la scelta di tecniche di intervento appropriate atte a ridurre il rischio per l'ambiente e la salute pubblica richiedono dettagliate valutazioni sito specifiche finalizzate ad indicarne la fattibilità e la sostenibilità ambientale, economica e tecnica. ${ }^{4}$

Le tecniche di incapsulamento sono tecniche di isolamento superficiale, isolamento perimetrale ed isolamento di fondo del sito contaminato. Per 


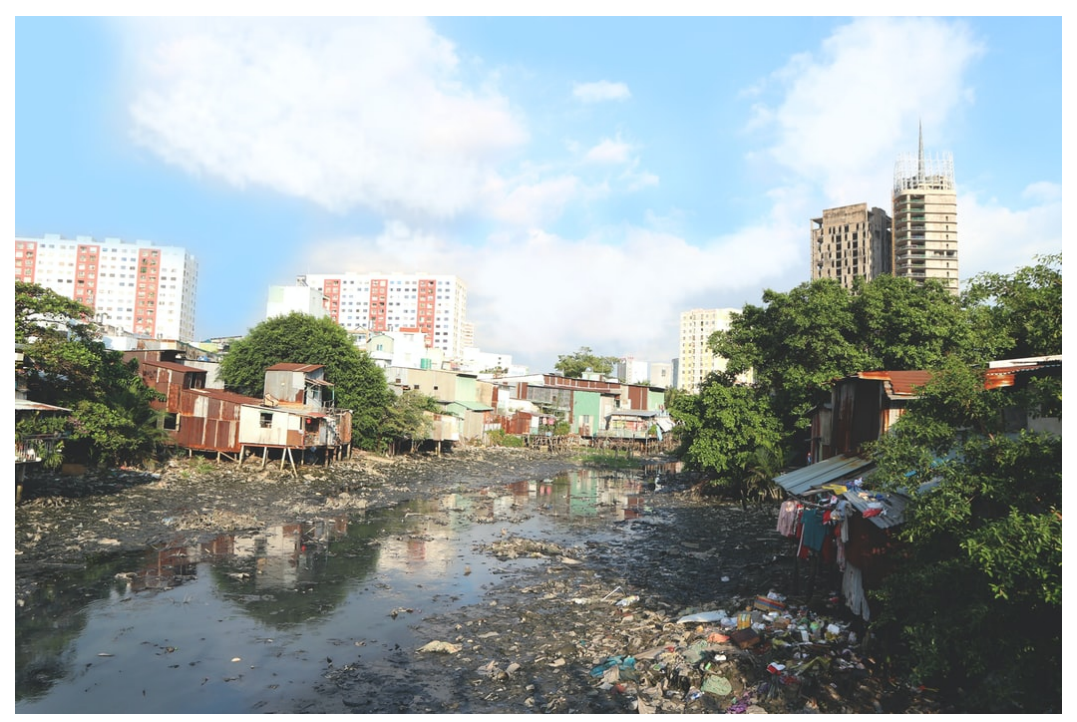

Figure 1: Sito Inquinato

quanto riguarda l'isolamento superficiale, questi metodi sono rivolti al contenimento del sito mediante barriere impermeabili multistrato o altro materiale, che impediscono la percolazione delle acque superficiali. ${ }^{5}$

Le barriere perimetrali possono essere passive o attive, e la protezione del suolo, delle acque freatiche e delle acque superficiali deve essere realizzata mediante la combinazione di una barriera geologica e di un eventuale rivestimento della parte inferiore attraverso il completamento della stessa con un sistema barriera di confinamento. ${ }^{6}$

I diversi sistemi utilizzabili possono essere classificati in base alla configurazione d'utilizzo o ai sistemi costruttivi. In base alla Configurazione d'Utilizzo, possiamo far riferimento a due tipologie di sistemazione, quella altimetrica e quella perimetrale.

La Sistemazione Altimetrica consiste nell'inserimento di un diaframma in posizione verticale con lo scopo di isolare la zona acquifera inquinata in modo da contenere i contaminanti. II diaframma immerso funge da barriera di protezione e viene inserito fin dentro il sottostante substrato impermeabile. $\mathrm{Nel}$ caso il contaminante sia di bassa densità e immiscibile in acqua viene inserito un diaframma sospeso nel terreno in modo da isolare soltanto la parte superficiale dell'acquifero.

Nel caso di Sistemazione Planimetrica si distinguono tre tipologie di cinturazione, la cinturazione parziale a monte della zona inquinata, la cinturazione parziale a valle della zona inquinata e la cinturazione completa. ${ }^{7}$

Per quanto riguarda i Sistemi Costruttivi utilizzati, si distinguono tre ti- 
pi diversi di barriere. Le barriere ad infissione consistono nell'infissione nel terreno di elementi metallici o prefabbricati in calcestruzzo. Tra questi, si trovano diverse tipologie che possono essere palizzate in acciaio, diaframmi a trave infissa e diaframmi a moduli di acciaio infissi. D'altra parte, le barriere per sostituzione consistono nella riduzione della permeabilità in sito attraverso l'introduzione nel terreno di miscele impermeabilizzanti. La tecnica più utilizzata è il Jet-grouting. Invece, le barriere per escavazione prevedono due fasi quali si realizza prima lo scavo della trincea con l'ausilio di una miscela di sostegno che evita il cedimento dello scavo e poi la sostituzione della miscela di sostegno con quella impermeabilizzante. Si distinguono due tipi di barriere che possono essere diaframmi plastici, tra i quali comprendono sistemi terreno-bentonite, cemento-bentonite, in calcestruzzo plastico e plastici sottili, oppure diaframma composito.

L'ultima modalità di contenimento trattata è l'Isolamento di fondo. Questi sistemi di isolamento permettono di impermeabilizzare il fondo della zona contaminata attraverso due tipologie di processi, un processo di sostituzione (o iniezione), e un processo di congelamento ${ }^{7}$. Per le barriere di fondo si utilizzano tecniche di iniezione Jet-grouting, iniezioni per frattura idraulica o per permeazione, e tecniche minerarie di asportazione e sostituzione del terreno.

Per ultimo, gli interventi di messa in sicurezza di fissaggio o immobilizzazione mirano a ridurre la mobilità dei contaminanti tra le matrici ambientali. Sono principalmente tecniche di solidificazione/ stabilizzazione, di vetrificazione e di termodistruzione ${ }^{8}$. In generale, le tecniche di fissaggio si utilizzano in tanto quando non è possibile utilizzare in tale area ulteriore tecniche di messa in sicurezza o di bonifica, a differenza delle tecniche di incapsulamento preliminare.

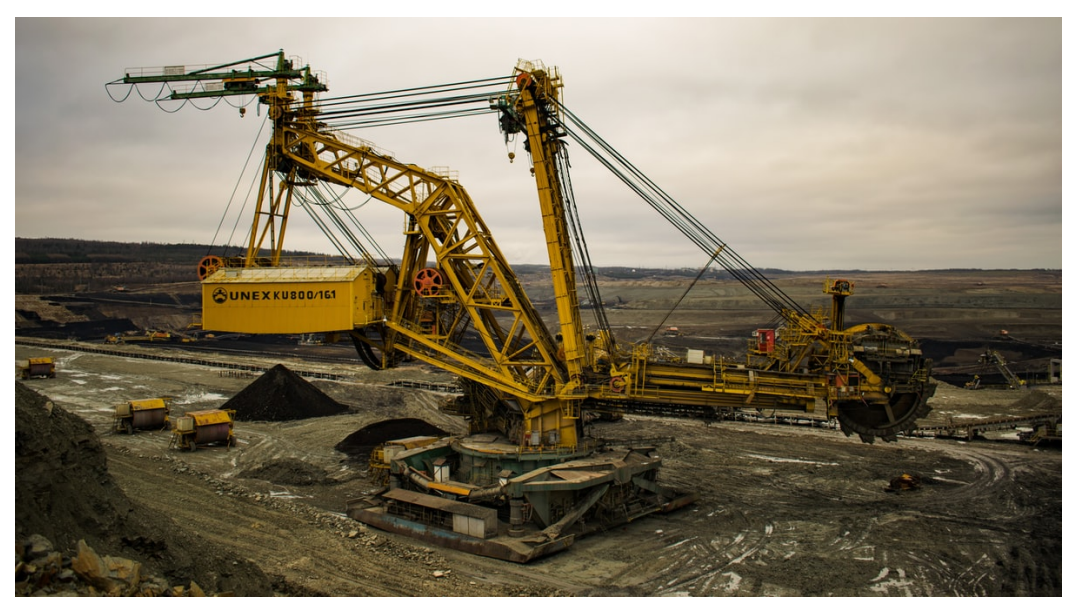

Figure 2: Sistemi Costruttivi: Barriere 


\section{Normativa di riferimento del caso studio}

La procedura effettuata nell'analisi del caso studio corrisponde alle linee guide "Land Contamination: Risk Management" (Gestione dei Rischi di Siti Contaminati), requisiti dell'Environment Agency del Regno Unito ${ }^{9}$. L'Agenzia per l'Ambiente è un ente pubblico non dipartimentale, istituito nel 1995 e patrocinato dal Department for Environment, Food and Rural Affairs (DEFRA) del governo del Regno Unito, con responsabilità in materia di protezione e valorizzazione dell'ambiente in Inghilterra. ${ }^{10}$

Gli step da seguire sono i seguenti:

- Valutazione dei rischi, la quale comprende la valutazione preliminare, quantitativa generica e quantitativa dettagliata del rischio.

- Valutazione delle opzioni tra cui è necessario decidere se sono disponibili informazioni sufficienti. Individuare possibili opzioni di riparazione in cui bisogna effettuare una valutazione dettagliata delle opzioni e selezionare le opzioni di riparazione finale.

- II Risanamento consente di elaborare non solo una strategia di risanamento, bonifica e verifica, ma anche monitoraggio e manutenzione a lungo termine.

- L'indagine sul sito consta dell'indagine intrusiva sul sito. Occorre considerare praticabilità, costi, efficacia e benefici insieme alle prove chimiche sui terreni.

- Obblighi di segnalazione attraverso rapporti di ciascuna delle fasi precedenti.

\section{Caso studio: sistema di contenimento attivo per un ex sito industriale nella zona est di Londra}

II sito in questione si trova in un'area altamente industriale nella zona est di Londra che comprende una superficie di circa 0,3 ettari, dove è presente un'ampia e variegata contaminazione in diverse aree interne a seguito di attività storiche e attuali. Questa condizione è aggravata dall'elevata sensibilità delle acque sotterranee e della presenza del fiume Lea lungo il confine orientale del sito.

Prima di scegliere un'adeguata opzione di risanamento, è stata intrapresa una valutazione del rischio qualitativo basata su un modello sorgente- 
percorso-bersaglio per valutare i rischi individuati durante le indagini intrusive. Da questa valutazione dei rischi è emerso che i suoli presenti nel sito rappresentavano un rischio elevato per i futuri utilizzatori del sito e per i lavoratori edili attraverso l'ingestione, l'inalazione e l'esposizione cutanea.

La scelta della strategia di risanamento ottimale deve considerare una serie di fattori, quali: lo spazio disponibile limitato, sistema stradale locale congestionato, futura implementazione della Direttiva sulle Discariche, tempi di sviluppo e budget, requisiti dell'agenzia per l'ambiente, dell'autorità locale e del servizio pubblico, problemi di sostenibilità, presenza di due acqua poco profonda di grande diametro che alimentano il sito e un grado di rischio residuo accettabile/potenziale di responsabilità. Sulla base delle considerazioni precedenti si è considerato che la soluzione di Dig and Dump non fosse economicamente sostenibile. L'inadeguatezza, le restrizioni di spazio e un'elevata falda freatica hanno precluso l'uso del trattamento ex situ dell'intero sito. Inoltre, sulla base dei risultati delle indagini in campo, si è ritenuto che la soluzione di risanamento economicamente ed ambientalmente più sostenibile per il sito fosse l'uso di un Sistema di Contenimento Attivo (Active Containment System) sotto forma di tagliafuori muratura nelle profonde argille impermeabili del Lambeth Group. Questo sistema è illustrato nella (Fig. 3).

Figure 3: Dettagli del sistema di contenimento attivo (ACS)

La metodologia di messa in sicurezza per il sito è quella di una barriera verticale a terra che comprende sezioni impermeabili passive e sezioni attive permeabili che si estendono per almeno $0,5 \mathrm{~m}$ nelle argille effettivamente impermeabili del Lambeth Group.

Le misure di protezione dai gas sono proposte anche all'interno dell'edificio e comprendono la fornitura di una membrana resistente ai gas e di una ventilazione passiva mediante geocomposito e cassette di sfiato. Si propone che le sezioni passive impermeabili siano costituite da pali di lamiera di acciaio sigillati e da una parete di fanghi di bentonite per accelerare i tempi di costruzione. Inoltre, saranno utilizzate lunghezze limitate di cemento bentonite coclea a volo continuo (CFA) per formare il collegamento tra le sezioni passive e quelle attive e verrà utilizzata una parete portante di pali con diametro di $900 \mathrm{~mm}$ sovrapposti. Infine, si conclude che la soluzione più efficiente e meno invasiva in tali aree potrebbe essere raggiunta utilizzando jet grouting. Le sezioni attive permeabili saranno installate a monte e a valle e comprenderanno una parete di cumulo di argilla attiva seccante CFA e il reagente attivo nelle sezioni attive utilizzato sarebbe E-Clay. ${ }^{11}$ 


\section{Conclusione}

L'elevata complessità del sito, dovuta all'ampia e variegata contaminazione del suolo e delle acque sotterranee, alle sue dimensioni, alla vicinanza ai recettori locali e ai servizi, renderebbe antieconomico un approccio convenzione di bonifica. In accordo alla normativa italiana, D.Igs. 152/2006 sull'individuazione delle migliori tecniche di intervento a costi sostenibili, l'utilizzo di ACS è risultata la migliore scelta. Sebbene ci siano diverse opzioni di interventi di messa in sicurezza attualmente disponibili, rende utile capire quali sono i benefici e svantaggi non solo dell'impiego e la disponibilità di questi ma anche delle caratteristiche del sito inquinato. Per concludere, è stato evidenziato l'impiego di alcune delle tecniche di messa in sicurezza di cui sopra menzionate in un caso studio e i principali criteri elencati dalla normativa di riferimento relativa a questi interventi.

\section{References}

1.A. Veneto - Tipologie di Intervento.

2.T.U. Ambiente - D.lgs. n. 152/2006.

3.DIgs 36/2003.

4.SEED Ricerca - MESSA IN SICUREZZA O BONIFICA.

5.IFRAS, A. I.-. Linee guida per la redazione dei progetti e la realizzazione degli interventi di bonifica e risanamento ambientale delle Aree Minerarie Dismesse. (2005)

6.SEED Ricerca - MESSA IN SICUREZZA O BONIFICA.

7.Contenimento fisico - Wikipedia.

8.Enrico, B., Carmine, M., Felice, U. \& Giovanni, Z. Tecnologie di bonifica dei siti contaminati: Principi di funzionamento ed esempi di applicazione. (Cacucci, 2014).

9.Land contamination risk management (LCRM).

10.Environment Agency - Wikipedia.

11.Swords, C. \& Strange, J. Active containment system for a former industrial site in East London. Engineering Geology 85, 204-211 (2006). 